As a conclusion we shall comment upon phenomena IV, the 'unseen mechanical energy' which certainly exists besides this part of the $V$-field detected by lines wiggles.

Unpublished estimates of the smoothing of the macro-turbulent $V$-field by insufficient resolving power, suggest that the true macroscopic motions $\xi^{2}$ are only a factor of 3 to 5 higher than observed ( $\mathrm{I} \cdot 7$ to 2.3 on $\xi$ ). Therefore the macroscopic motions remain smaller by a factor of 3 or more, than the turbulent velocities derived from the curve of growth and profiles. The motions involving optically thin volume elements are accordingly the essential part of the mechanical energy in the solar stmosphere.

According to Osterbrock, the acoustical noise spectrum from the deep convection zone might be peaked around $\nu_{0}=\mathrm{I} \cdot 2 \times 10^{-2} \mathrm{~Hz}(t=80 \mathrm{sec})$. Sound waves at this supposed peak and higher frequencies have a wavelength $\Lambda$ of $500 \mathrm{~km}$ and smaller and cannot give observable line shifts, because the thickness of line formation layers extends on a length which is a large fraction of $\Lambda$. Also we may expect the extent of the fronts of such short wave elements to be small (say of the order of $\Lambda$ ) and below the possibility of resolution. So we suggest that the microscopic $V$-field transported through the atmosphere (and mainly responsible for coronal heating) is high frequency, that is acoustical noise around $10^{-2} \mathrm{~Hz}$.

\title{
DISCUSSION
}

R. Michard (answering questions by Dr Giovanelli and Dr Rösch). Concerning the sketch summarizing the 'supergranulation', I completely agree that his picture is still tentative. In particular it is not proved that the pattern of downwards flow in $\mathrm{H} \alpha$ is related to the supergranulation.

F. Rösch. It is possible that changes in the brightness-velocity correlation for photospheric granules will occur when the image resolution is increased.

\section{THE SOLAR HYDROGEN CONVECTION ZONE AND ITS DIRECT INFLUENCE ON THE PHOTOSPHERE}

\author{
E. A. Spiegel \\ (Physics Department, New York University)
}

The diversity of phenomena in the solar atmosphere, which observers have been discovering at a dramatically accelerated pace in recent years, seems to defy all attempts at a general chacterization. Nevertheless, it seems clear that the energy implied by the observed inhomogeneities, velocity fields, and magnetic fields, is derived from the motions originating in the solar hydrogen convection zone. Thus, for a complete understanding of solar phenomena we certainly require not only a theory of convective motion, but also an understanding of the couplings among the various modes of energy which are observed or inferred. Neither of these is really available, in spite of some recent progress in the understanding of laboratory convection, and it is likely that the two problems can only be solved together. The upshot is that a gap exists between the present exciting picture provided by solar observers and the type of phenomenon that the theory of convection can even clarify qualitatively. In spite of this very real separation between the realms of observational and theoretical knowledge a discussion of the interaction between the convection zone and the photosphere may be useful at this time, if only to illustrate the present possibilities for theoretical interpretation. 
To proceed usefully, it is necessary to limit severely the range of solar phenomona to be discussed and to include only those which seem to be direct manifestations of convective motion. In doing this we avoid the problems of coupling between convective motion and induced phenomena. I hope I am not being too conservative, then, if I restrict myself here to a discussion of the relation of convection theory to the granulation and supergranulation. Such a discussion normally proceeds in two steps:

I. Calculate the mean structure of the convection zone (and the overlying atmosphere) using the mixing length theory.

2. Study the dynamical behavior of fluctuations imposed on this mean structure to get some idea of the kind of time scales and length scales which may be important.

Now these two steps are strictly not independent since the convective heat transfer is an average of the product of the fluctuating temperature and vertical velocities. However, the approximations of the mixing length theory, though crude, permit the separation of the two calculations. We need not dwell on Step I here since it is the topic which Mrs Böhm will deal with in her report.

Step 2 proceeds along the following lines. One considers the equations of motion for a disturbance of infinitesimal amplitude applied to the steady-state configuration of Step I. The assumption of small amplitude leads to formally linear equations which are separable in space and time. If the convection zone is plane-parallel, a typical fluctuation quantity has solutions of the form

$$
w(x, y, z, t)=\mathrm{e}^{\eta t+i l x+i m y} W(z)
$$

where $z$ is the vertical co-ordinate, $x$ and $y$ are the horizontal co-ordinates, and $W$ satisfies an ordinary differential equation. In this equation the separation constant $\eta$ appears as an eigenvalue parameter and $k=\sqrt{l^{2}+m^{2}}$ appears as a free parameter. The length, $\pi / k$, characterizes the horizontal scale of the impressed disturbance and the inverse time, $\eta$, is known as the growth rate, though it may be complex or imaginary. Of course, boundary conditions must be prescribed but I shall not go into this delicate question here.

The main aim of the calculations just described has been to find how the growth rate, $\eta$, depends on $k$. For convectively unstable layers, $\eta$ has real positive values, and the feeling has been that the $k$ for which $\eta$ is maximum should give some indication of the scale of motion which is dominant in solar convection. However, $\eta$ cannot give any indication whatever of the relative importance of different scales, since the linear equations contain no information about the direct couplings among various scales of motion. But it must certainly be true that such couplings play an important role in determining relative amplitudes.

Though the calculation of the convective growth rate is as $I$ have outlined it straightforward in principle, it is very difficult in practice. Even the linear equations must adequately describe the effects of strong density variation, radiative transfer and changes of stability conditions across the medium. Hence the calculations of $\eta$ have been performed principally for idealized models of the convective layer and it is on such work that most of our present understanding depends. The literature of these calculations is becoming extensive and cannot be reviewed here, but it contains such names as Böhm, Richter, Skumanich, and Unno, among others. What this literature reveals is that, in the absence of dissipative mechanisms, $\eta$ is a monotonically increasing function of $k$, quite irrespective of the details of the underlying mean state.

The principle observational facts which these calculations are intended to clarify are the observations of time-dependent inhomogeneities in the solar photosphere, as exemplified by the spectrum of brightness fluctuations observed in the Stratoscope balloon observations (r). As a function of $k$, the spectrum rises from zero at $k=0$ to a maximum at $\pi / k \approx 10^{3} \mathrm{~km}$ and cuts off in the neighborhood of $\pi / k \approx 250 \mathrm{~km}$. Now this cutoff is close to the resolution limit 
of the Stratoscope telescope, but Bahng and Schwarzschild have argued that the actual spectrum does drop off rapidly near this limit. If this is the case, then we must explain why the small scale motions, which are unstable, do not show up in the photospheric observations.

One suggested explanation has been that radiative damping can provide a cutoff analogous to that observed. Indeed, in his calculation of $\eta$, based on Mrs Böhm's accurate model, Böhm (2) has included radiative transfer and has obtained a radiative cutoff. But this cutoff is at a much larger value of $k$ than the observed one. Moreover, Böhm has used an Eddington approximation for the radiative damping at large $k$. Indeed, it appears that with a more accurate treatment of the radiation terms, no cutoff occurs until $k$ is so large that atomic conduction is important (3). Thus, there is here an apparently great discrepancy between theory and observation.

A possible resolution of the discrepancy may lie in the different amplitude distributions of modes of different $k$. For it must be stressed that a positive value of $\eta$ means only that the mode is unstable globally, even though the local stability conditions may vary greatly. For a particular mode of wavenumber $k$, the local stability is measured by the Rayleigh number based on $k$. This Rayleigh number is just the local value of the ratio of the bouyancy force to the viscous force, and it is only where this ratio is greater than unity can we expect the driving forces to do net work on the disturbance. More precisely, the local criterion for instability (based also on an Eddington approximation) is

$$
\left(\frac{\mathrm{I} 6 \sigma}{3 g}\right) \frac{\mu T^{3} H_{p} k^{4}}{\kappa C_{p}|\Delta \nabla| \rho^{3}} \leqslant \mathrm{I},
$$

where $\mu$ is the viscosity, $\sigma$ the Stefan-Boltzmann constant, and the other symbols are standard. Where this criterion is satisfied, the mode will have large amplitude; elsewhere it will have small amplitude. Since $\rho$ is small at the top of the convection zone, small scale motions ( $k$ large) will have small amplitude there even though the gradient is superadiabatic. The equality in (2) defines the transition point above which a mode will have small amplitude and permits us to determine what scales of convective motion should be detectable.

With the cooperation of Miss Hofmeister and Dr Weigert, and with values obtained from their solar model (4), it has been possible to determine for each $k$ the optical depth, $\tau$, of the transition point from large to small amplitude. Space is not available here to display these results; but they reveal that horizontal scales smaller than $210 \mathrm{~km}$ should have small amplitude for $\tau \leqslant \mathrm{I}$. Thus though smaller scales must be quite unstable they just cannot be seen from the outside in the form of convective motions. This means that only larger scale convective motions make their way undeterred to the top of the convection zone, and possibly beyond. Thus the largest permitted scale of motion should be quite prominent; these are scales comparable to the thickness of the convection zone. The phenomenon of supergranulation seems to fit this description very well. Indeed, the scales of motion observed in the supergranulation apparently provide an observational confirmation of the estimated thickness of the convection zone.

Since the amplitude distributions for these largest scales of motion are not affected by dissipative processes, they probably are governed chiefly by the constraint of mass conservation. This remark is based on the linear calculations which omit dissipation. For the flow in a large, but transient, convective cell to conserve mass, it is necessary for the velocity to be large where the density is small. This results in a rapid horizontal streaming concentrated in about the uppermost density scale height of the convection zone. This horizontal flow is analogous to the flow observed near the rigid boundaries of laboratory convection experiments (5). Kraichnan (6) has suggested that such a flow against a boundary should produce a shear instability and one may wonder whether the flow at the top of the convection zone may also be unstable. An estimate of the local Reynolds number reveals that it is. Of course, this instability is just a 
manifestation of the variety of phenomena which are implicitly described by the nonlinear terms discarded in Step 2, and we are here just qualitatively reconstructing part of what was lost in the linearization. What we can expect is the occurrence of turbulent motion predominantly of scales of several hundred kilometers. This turbulence is mechanically driven, but as it is produced in a convectively unstable region, a positive correlation between temperature and velocity will be impressed on it. Moreover, it will probably generate a random, small scale magnetic field with the magnetic energies equal to the kinetic energies.

From this discussion there emerges a picture which suggests two dominant observable scales of motion. The first is the large scale motion which reflects the pattern of overall convective motions through the main body of the convection zone. Though smaller scale convective motions occur, they do not have large amplitude near the top of the zone, and so the observable spectrum of scales of motion drops off from a maximum at scales of tens of thousands of kilometers. This theoretical maximum should be associated with supergranulation. As smaller scales are approached, a secondary maximum will appear at scales of several hundred to rooo kilometers. This secondary maximum represents motions driven mechanically rather than thermally, and may account in some measure for the ordinary granulation.

Unfortunately, space limitations permit only this sketchy description here and do not permit details such as acoustic behavior to be discussed. But I should like in conclusion, to allude to one possible aspect of the penetration of motion from the unstable zone into the overlying unstable layers. Astrophysical studies, e.g. Unno (7) indicate that large scale motions will penetrate deeply into the stable regions. But these (justifiably) do not include viscous effects. When viscosity is included, the indications are that the motion does not penetrate deeply, but instead produces a countercell in the overlying stable layer (8). Experiments at Woods Hole by Rooth and Furumoto show that this countercell has small vertical extent, but has a horizontal structure like that of the underlying driving motion. Now in the astrophysical case, the small scale motion I have described will provide an eddy viscosity and may couple the stable and unstable layers in the way that ordinary viscosity does in the laboratory. We must then consider the possiblity that the large scale solar convective motion may drive countercells rather than penetrate into the stable regions. These countercells will have horizontal dimension comparable to the supergranulation, and it may be these, rather than the actual cellular motion, which is observed directly. But this is a tentative suggestion whose main purpose here is to illustrate the kinds of possible bearing present theoretical results on convection may have on interpretations of solar observations.

I should like to express my gratitude to the Air Force Office of Scientific Research for support during the preparation of this report under Contract no. AF-AFOSR-62-386. I am also grateful to the Max-Planck Institut für Astrophysik for its hospitality during which some of the reported results were calculated, and especially to Dr Weigert, Dr Kippenhahn and Miss Hofmeister for the stimulating atmosphere they provided.

\section{REFERENCES}

I. Bahng, J. D., Schwarzschild, M. Astrophys Y., 134, 312, x96r.

2. Böhm, K. H. Astrophys. F., 137, 881, I963.

3. Spiegel, E. A. Astrophys. F., 139, 959, 1964.

4. Hofmeister, E., Weigert, A. Z. Astrophys., 59, I 19, 1964.

5. Malkus, W. V. R. Proc. R. Soc. Lond., (A) 225, $185,1954$.

6. Kraichnan R. H. Phys. of Fluids, 5, I 374, 1962.

7. Unno, W. Astrophys. F., 126, 259, 1957.

8. Veronis, G. Astrophys. $\mathscr{F} ., 137,641,1963$. 